\title{
BMJ Open Deprescribing in primary care in Portugal (DePil17-20): a three-phase observational and experimental study protocol
}

Pedro Augusto Simões, ${ }^{1,2}$ Luiz Miguel Santiago, ${ }^{3,4}$ José Augusto Simões ${ }^{1,5,6}$

To cite: Simões PA, Santiago LM, Simões JA. Deprescribing in primary care in Portugal (DePil17-20): a three-phase observational and experimental study protocol. BMJ Open 2018;8:e019542. doi:10.1136/ bmjopen-2017-019542

- Prepublication history for this paper is available online. To view these files, please visit the journal online (http://dx.doi. org/10.1136/bmjopen-2017019542).

Received 12 November 2017

Revised 9 May 2018

Accepted 7 June 2018

D Check for updates

(c) Author(s) (or their employer(s)) 2018. Re-use permitted under CC BY-NC. No commercial re-use. See rights and permissions. Published by BMJ.

${ }^{1}$ Faculty of Health Sciences, University of Beira Interior, Covilhã, Portugal

${ }^{2}$ USF Pulsar, ARS Centro, Coimbra, Portugal

${ }^{3}$ Faculty of Medicine, University of Coimbra, Coimbra, Portugal ${ }^{4}$ USF Topázio, ARS Centro, Coimbra, Portugal ${ }^{5}$ UCSP Mealhada, ARS Centro, Mealhada, Portugal

${ }^{6}$ CINTESIS - Centre for Research in Health Technologies and Service, Porto, Portugal

Correspondence to MD Pedro Augusto Simões; pedro.augusto.simoes@ubi.pt

\section{ABSTRACT}

Introduction Polypharmacy is commonly defined as the simultaneous taking of five or more drugs. Deprescribing is the process of tapering or stopping medications with the aim of improving patient outcomes and optimising current therapy, and there are several tools aiming at identifying potentially inappropriate medications, especially in the elderly. The direct involvement of patients and their caregivers in the choice and administration of drugs has long been known to be very important, but it is not usually applied. The aim of this study is to assess the knowledge of older adults about deprescription, the effect on willingness to have regular medications deprescribed and its quality-of-life outcome.

Methods and analysis This study protocol comprises three phases. The first two phases will be nationwide and aim to evaluate the prevalence and patterns of polypharmacy and assess the barriers and facilitators of deprescribing perceived by older adults, as well as their willingness to have regular medications deprescribed and to self-medicate. The third and last phase will be a nonpharmacological randomised clinical study to measure older patients' acceptance to have regular medications deprescribed and related quality of life.

Ethics and dissemination The study will be conducted in accordance with the principles expressed in the Declaration of Helsinki. It has been approved by the Ethics Committee of the University of Beira Interior and Portuguese National Data Protection Commission. Study results will be published in peer-reviewed journals and presented at national and international conferences. In short, no action will be taken without written consent from patients and doctors.

Trial registration number >NCT03283735.

\section{INTRODUCTION}

Polypharmacy is commonly defined as the simultaneous taking of five or more drugs, ${ }^{1}$ but it can also be defined as using medication that is not indicated, not effective or is therapeutic duplication. ${ }^{2}$ It is present in $30 \%-70 \%$ of older adults ${ }^{3}$ and it is a significant predictor of the risk of falls, ${ }^{4}$ inappropriate prescriptions, reduced patient adherence, drug interactions, hospital admissions ${ }^{56}$ and mortality. ${ }^{7}$
Strengths and limitations of this study

- Phase I and II will be nationwide and will be the first ones to take place in Portugal.

- This will be one of the first to assess the impact of deprescribing in health and quality-of-life outcomes on older adults.

- Study methodology comprehensively aims at getting the whole picture of the problem from its epidemiological study, through the understanding of what polymedicated patients feel about being on less medication load, until the perception of quality-of-life study when deprescription has been made.

- The relatively small sample will be a methodological limitation because it will not allow getting so strong conclusions as if the sample was bigger, due to medical short adherence because of the workload.

- The possible contamination of the intervention in phase III, due to parallel sources of information, taking place as a confounding variable.

It is estimated that at least $75 \%$ of this adverse event is potentially preventable. ${ }^{8}$

Potentially inappropriate medications (PIM) are those for which the harms outweigh the benefits, namely those that are not indicated or lack evidence of efficacy and those that do not align with patients goals/ preference and values. ${ }^{9} \mathrm{So}$, it is necessary to distinguish between appropriate and inappropriate medications ${ }^{10}$ because as people get older the benefit:risk ratio of medications changes, meaning that medications that were once appropriately prescribed may have become inappropriate. ${ }^{11}$ An Australian study reported that $60 \%$ patients had at least one PIM, leading to a high risk of adverse drug reactions, morbidity and mortality. ${ }^{12}$ There are a lot of guidelines about when to start medication that is safe and effective, but there is a lack of similar guidelines for ceasing inappropriate medication. ${ }^{13}$

Deprescribing is the process of tapering or stopping medications with the aim of 
improving patient outcomes and optimising current therapy. ${ }^{14}$ However, it is not free of risks, namely withdrawal syndromes, rebound effects, pharmacokinetic/ pharmacodynamic changes in the remaining drugs and recurrence of the symptoms. ${ }^{315}$ So, the decision to deprescribe results from a careful weighting between the therapeutic objectives and the risk:benefit ratio.

Many deprescribing processes have been proposed in the literature. ${ }^{1516}$ One of the most widely used is a simple five-step protocol consisting of a comprehensive medication history, identifying PIM (attending to the harms and benefits of medication, as well as to the life expectancy and care goals), determining whether medication can be ceased and prioritisation (taking into account the patient's preferences), planning and initiating medication withdrawal (one at a time and often with tapering) and close monitoring and documenting the improvement in health and quality of life and the reduction of adverse effects. ${ }^{17}$

Almost a dozen medication screening tools exist in order to aid identifying PIM in older adults and improve their care. The most widely used are Beers criteria and the Screening Tool of Older Person's Prescriptions and Screening Tool to Alert Doctors to Right Treatment (STOPP/START) criteria. Both the Beers criteria and the STOPP component of the STOPP/START criteria are lists of medications that should be avoided in older adults because of its adverse effects and drug-drug and drug-disease interactions. On the other hand, the START component of the STOPP/START criteria consists of a list of medications that should be considered to initiate in the presence of certain conditions. Another useful tool is the Medication Appropriateness Index that consist of issues to be taken into account before prescribing a medication. ${ }^{18}$

Many studies have recognised that the implementation of a deprescribing process is feasible in practice and acceptable to participants ${ }^{19} 20$ and, hypothetically, may result in favourable patient health and quality-of-life outcomes ${ }^{21}$; further studies are needed to confirm it. There are already a few number of strategies that appear to be effective and promising, ${ }^{22}$ however assessing the effectiveness of these interventions is difficult because different studies have different study designs, settings and types of interventions. Many of these studies have short follow-up periods (2 months to 1 year), so they may not provide information about the long-term impact of these interventions, and/or lack of clinical outcome measurements. ${ }^{23}$ One outcome measurement rarely used was the effect on health-related quality of life.

Patients are uncertain about their willingness to have a medication deprescribed because they are confused by conflicting advice on benefit and harm from different healthcare professionals. ${ }^{15}$ The majority of patients want to be involved in the decision-making process, ${ }^{17} 24$ and this has long been known to be very important, but shared decision-making is not routine. ${ }^{25}$ It is assumed that older people generally consider they take a lot of medications and complain about it, but they are reluctant to cease specific medications in practice. ${ }^{26}{ }^{27}$ So it is important to understand this incongruity between not liking to take multiple medications and reluctance to accept the proposal to stop them. In particular for Portuguese context, there are no studies on these matters, so it is necessary to understand such ambivalence, because it will help us solve many problems arising from polypharmacy, such as adverse drug reactions. ${ }^{28}$

There are only some studies about the prevalence of polypharmacy in some region of Portugal, none nationwide. Also, there are no studies about the Portuguese older adults' attitudes and beliefs regarding medication, and there are very few studies around the world. Finally, most of the studies focus on the effect of deprescribing in clinical outcomes such as falls, consultations rates, hospitalisations and/or mortality. Very few focus on the effect on quality of life and older adults' willingness. In order to study the phenomenon, as well as to create rationales, this work is necessary.

\section{Terminology}

For the purpose of defining polypharmacy, we will use the list of active ingredient of drugs and consider three definitions: $\geq 5$ drugs versus $\geq$ the median number of drugs versus presence of at least one PIM. The rationale for such resides in the scarcity of studies on the number of medications simultaneously taken. In fact, due to multimorbidity, many elderly patients are taking more and more drugs. ${ }^{29}$ So, we want to compare the international accepted definition ( $\geq 5$ drugs) with this new approach to see if there are differences.

\section{Study objectives}

The primary objective is to assess the knowledge of older adults about deprescription, the effect on willingness to have regular medications deprescribed and their quality-of-life outcome.

Specific objectives are:

- To identify the prevalence of polypharmacy in older adults in Portugal.

- To evaluate the proportion of PIM in older adults in Portugal.

- To describe the sociodemographic and clinical profiles of older adults with polypharmacy in Portugal.

- To identify the main barriers to and the facilitators of deprescribing in Portuguese older adults.

- To evaluate the Portuguese older adults willingness to have regular medications deprescribed.

- To correlate the self-medication with the willingness to have regular medications deprescribed.

- To evaluate the effect in quality of life after having regular medications deprescribed.

- To elaborate and validate a flow chart with the deprescribing process, in the patient's perspective. 


\section{METHODS AND ANALYSIS}

\section{Study design}

This is a three-phase study:

1. Cross-sectional, analytical study of the prevalence and patterns of polypharmacy, namely sociodemographic and clinical profiles (age, gender, area of residence and years of study) and about medication (number of drugs and their active component), in older adults attending primary care in Portugal.

2. Cross-sectional, triangulation study of older adults' perception of barriers to and facilitators of deprescribing, willingness to have regular medications deprescribed and willingness to self-medicate.

3. Non-pharmacological randomised clinical study of older patients' acceptance to have regular medications deprescribed and related quality of life.

\section{Phase I}

Objectives

To assert the prevalence of polypharmacy in older adults attending primary care in Portugal and describe their sociodemographic and clinical profiles.

\section{Design}

Cross-sectional, analytical study.

\section{Setting}

Primary care centres in Portugal will be randomly selected from the five mainland Portuguese healthcare administrative regions and two autonomous regions (Madeira and Azores), in order to obtain a national geographical representative sample.

\section{Sample size}

Since the prevalence of polypharmacy in older adults is unknown, we will use as base of population all older adults in Portugal. For the study, we will use a 95\% CI and a maximum precision error of $5 \%$. According to Pordata (www.pordata.pt), the population of Portugal is around 10.33 million, of which 2.18 million are over the age of 65 . Since the literature suggests that the range of polypharmacy is $30 \%-70 \%$ and we think that it is over $50 \%$, we estimate that we would need at least 742 patients.

\section{Study procedures}

This phase of the study will start in March 2018.

We will ask the information department of the ministry of health for the data of 757 randomised patients (electronically stored): 245 in North of Portugal, 190 in Centre of Portugal, 211 in Lisbon-Tejo Valley, 65 in Alentejo, 33 in Algarve, 6 in Azores and 7 in Madeira in accordance with the distribution of Portuguese old adult population ( $\geq 65$ years) in Portugal according to Pordata.

\section{Data collection}

The collection of data will occur in March 2018.

Data will be electronically stored in a database specifically designed for this study. It will be encrypted and password protected. Information will be treated in strict confidentiality to protect the privacy of the patients. The investigators will have no access to the data of the patients, except the one provided by the information department of the ministry of health.

\section{Statistical analysis}

A descriptive analysis of all study variables will be performed, namely the number of valid observations, mean $\pm \mathrm{SD}$, median and range for quantitative variables and absolute and relative frequencies for qualitative variables. Prevalence of polypharmacy (considering the three definitions) will be calculated together with corresponding 95\% CI. Moreover, the prevalence of polypharmacy will be estimated by subgroups, namely age, gender, residence area and formal education. Univariate analysis will be conducted to study the associations between those characteristics and polypharmacy using $\chi^{2}$ test (qualitative characteristics) or Student's t-test/Mann-Whitney $\mathrm{U}$ test (quantitative characteristics). Multiple logistic regressions will be carried out considering the presence of polypharmacy as the dependent variable and patients' characteristics as the independent variable in order to calculate the OR and corresponding 95\% CI. Total number of drugs taken by the patients and their pharmacological classes will also be summarised together with 95\% CI, and multiple regressions may be performed to analyse its association with patients' characteristics. All tests will be two-sided using a significance level of 0.05 . Statistical analysis will be conducted using SPSS V.23.0 or higher.

\section{Phase II}

Objectives

To determine older peoples' attitudes and beliefs regarding medication use and their willingness to have regular medications deprescribed.

\section{Design}

Cross-sectional, analytical study.

\section{Setting}

It will be the same as phase I.

\section{Sample size}

Since the prevalence of polypharmacy in older adults is unknown, we will consider that it is around $60 \%$ of the older adults' population. So, we need at least 385 patients with polypharmacy, to obtain a sample with a $95 \%$ CI and a maximum precision error of $5 \%$.

\section{Study procedures}

This phase of the study is expected to start in October 2018.

For general practitioners (GPs) sampling we used existing files of previous projects adherent GPs, in other epidemiological studies, in order to have an higher adherence rate. After the selection of GPs, those who agree to participate will recruit their own patients, after their consent. Assuming that a GP will be able to include at 
least six patients, a total of 65 GPs will be enrolled in the study: 21 in North of Portugal, 16 in Centre of Portugal, 18 in Lisbon-Tejo Valley, 5 in Alentejo, 3 in Algarve, 1 in Azores and 1 in Madeira in accordance with the distribution of Portuguese old adult population ( $\geq 65$ years) in Portugal according to Pordata (www.pordata.pt).

Enrolled GPs will be instructed to give the questionnaire and the informed consent to all older adults $(\geq 65$ years) patients, with polypharmacy, attending a primary care consultation during the period of study: we will randomise six consultation days for data collection. GPs will collect all necessary data about the patients who sign the informed consent and fill all questions of the questionnaire. After that, we will randomise the pool of data according to gender and region, in order to obtain a sample in accordance with Portuguese distribution of old adult population ( $\geq 65$ years).

GPs and patients willing to participate in the study must give written informed consent and present ability to comply with the study requirements.

Exclusion criteria will be: being acutely unwell in the last 3 weeks and refusal to participate.

\section{Data collection}

The collection of data will occur in October 2018.

GPs will be responsible for collecting all data about patients' sociodemographic characteristics, as well as morbidity and medication, during their consultations. Moreover, the perception of medication will be evaluated using Portuguese general Beliefs about Medicines Questionnaire (BMQ), the willingness to have regular medications deprescribed will be assessed with one open-question ('What do you think about withdrawing medication?'), to evaluate the qualitative knowledge about the patient's acceptance, and the need to self-medicate with over-the-counter medication will be evaluated with a Visual Analogue Scale (0-10) about the need to self-medicate and its justification.

Those who do not know how to write or read can choose someone they know (eg, a family member or a friend) to write the answer.

In case of less than $50 \%$ of answers of the open questions, two patient groups will be invited to make a focus group asserting reasons for accepting deprescribing.

Data will be electronically stored in a database specifically designed for this study using MS Excel 2010. It will be encrypted and password protected. Information will be treated in strict confidentiality to protect the privacy of patients. The investigators will have no access to the data of the patients. The only person to know who is being studied will be the GP.

Before the collection of data, there will be online reunions with the GPs participating in the study.

We have been authorised to use BMQ by the authors.

\section{Statistical analysis}

A descriptive analysis of all study variables will be performed, namely the number of valid observations, mean $\pm \mathrm{SD}$, median and range for quantitative variables and absolute and relative frequencies for qualitative variables. We will categorise the willingness to have regular medications deprescribed in two groups (high and low). The perception of medication, willingness to have regular medications deprescribed and need to self-medicate will be estimated by subgroups, namely age, gender, residence area and formal education. Univariate analysis will be conducted to study the associations between those characteristics and the perception of medication, willingness to have regular medications deprescribed and need to self-medicate using $\chi^{2}$ test (qualitative characteristics) or t-test/Mann-Whitney (quantitative characteristics). Multiple logistic regressions will be carried out considering the perception of medication, willingness to have regular medications deprescribed and need to self-medicate as the dependent variable and patients' characteristics as the independent variable in order to calculate the OR and corresponding 95\% CI. All tests will be two-sided, considering a significance level of 0.05 .

\section{Null hypothesis}

The people with more willingness to have their regular medications deprescribed believe that medications are harmful and overused by doctors.

The need to self-medicate is present in people with less fear of medication and less overuse belief.

People with polypharmacy see no or little harm in the medication and do not think they have polypharmacy.

\section{Phase III}

Objectives

To measure older patients' acceptance to have regular medications deprescribed and related quality of life.

\section{Design}

Non-pharmacological cluster randomised clinical study, intended to last for 6 months.

\section{Outcomes}

Primary outcome will be the quality of life.

Secondary outcome will be the willingness to have regular medications deprescribed.

\section{Setting}

Primary care centres in Portugal will be randomly selected from six health centres of Centre of Portugal (Aveiro, Castelo Branco, Coimbra, Guarda, Leiria and Viseu).

\section{Sample size}

Since the prevalence of polypharmacy in older adults in Centre of Portugal is unknown, we will consider that it is around $60 \%$ of the older adults' population in this region (around 520 000). So we need at least 380 patients with polypharmacy, to obtain a sample with a $95 \% \mathrm{CI}$ and a maximum precision error of $5 \%$. However, assuming a dropout rate of around $25 \%$, we will increase the required sample by $25 \%$ in order to compensate for dropouts, so we will need at least 474 patients with 
polypharmacy. Then we will create two groups with a minimum of 237 patients each (one will be the intervention group and the other the control).

\section{Study procedures}

This phase of the study is expected to start in September 2019 and will last for 6 months.

Again, GPs sampling will be made according to existing files and those who agree to participate will recruit their own patients, after their consent. Patients from previous phase can be enrolled. Assuming that a GP will be able to include at least 10 patients, a total of 48 GPs have to be enrolled in the study. Enrolled GPs will be instructed to invite all older adult ( $\geq 65$ years) patients with polypharmacy, attending the primary care consultation to participate in the study until we obtain the required sample size, they will be randomised as described below. The geographical areas of work, the districts, will be randomised for entry into exposed and unexposed groups, in order to minimise the contamination of the intervention that could happen if we use randomisation at patient level. The purpose is to have doctors performing only one task in each district. To make both groups as homogenous as possible, we will group similar districts in order for them to be in different branches of the study.

Patients willing to participate in the study must give written informed consent and present willingness and ability to comply with the study requirements. The patients' recruitment procedure will be the same as the one described for phase II.

Exclusion criteria: being acutely unwell in the last 3 weeks and refusal to participate.

Two groups will be created with a minimum of 237 patients each, one of which will be composed of patients from the regions of Aveiro, Coimbra and Guarda and the other from patients from the regions of Castelo Branco, Leiria and Viseu. In the intervention group, we will give empowerment tools and will talk with their GPs about how to approach the problem of polypharmacy and the control group will receive the usual care. The information given in the intervention group will result from the knowledge obtained in phase II, it will be compiled in small leaflets and other informational materials to be made according to the best practice, to be given and remembered at scheduled times to this group. To summarise, this information will be used to educate GPs about how to approach the issue of deprescribing and to provide material to participants, during a consult, so that they can learn more about it.

\section{Data collection}

The collection of data will occur in the beginning (baseline) and end of phase III (at 6 months), in order to analyse changes from baseline.

GPs will be responsible for collecting all data. Patient's sociodemographic and clinical characteristics, and medication will be registered using the same methodology as described in phase II.
Perception of medication will be evaluated using Portuguese general $\mathrm{BMQ}$, the willingness to have regular medications deprescribed will be assessed with one open question (the same as phase II), and the quality of life will be assessed with EuroQol Five Dimensions Questionnaire (EQ-5D), a validated tool for Portugal. The aim is to observe the impact of deprescription on health-related quality of life, even if, to our knowledge no study has used EQ-5D in this specific domain in Portugal.

Those who do not know how to write or read can choose someone they know (eg, a family member or a friend) to write the answer.

We have been authorised to use BMQ and EQ-5D by the authors.

\section{Statistical analysis}

It will be similar to phase II. Comparisons between baseline and the 6-month groups regarding a quantitative variable are to be made using t-test or Sign/Wilcoxon non-parametric test, if normality assumption is not met.

\section{Null hypothesis}

The intervention will result in higher quality of life.

\section{Patient and public involvement}

Patients and/or public were not involved.

\section{DISCUSSION}

This will be the first study to assess prevalence and patterns of polypharmacy in older adults in Portugal and one of the first to assess the impact of deprescribing in health and quality-of-life outcomes on older adults. We hope that the results will help clinicians to better understand patient's perception regarding polypharmacy and deprescribing.

However, many Portuguese GPs are not very keen to participate in studies like this because they have a heavy workload. Therefore, special attention and care are put on the size of the sample to achieve the goal of the study and in the recruitment methodology. Moreover, we will support and interact constantly with the participant GPs, in order to maintain their motivation. Because of this anticipated short adherence, we conclude that it would be impracticable to conduct phase III at the national level.

For phase III, a strategy was thought of trying to make geographical contamination as little as possible. Therefore we randomized the geographical areas instead of GPs or patients, so that GPs wouldn't discuss the intervention between themselves. As it is an area still unknown in Portugal, we will try to control external interventions about deprescription, but, of course, in a interconnected world where the news spreads via the internet, some contamination will surely happen and may be a confounding variable, but it will be a systematic one. Also, in order to make both groups as homogenous as possible, we will group similar, but apart, districts, where doctors will perform the tasks. GPs will be the focus of meetings 
and ongoing mails so that the study is completed. Since there is no access to the identification of patients, the most suitable way to conduct the study is through randomisation of patients by voluntary GPs. This will probably be a bias but, in light of the Portuguese laws, there is no other way to do it and, once again, there is no fee for task study. The fact that there is randomisation of patients, guaranteed by the size of the epidemiological representative samples, will provide a clear picture of the intended study problem.

\section{Ethics and dissemination}

The study will be conducted in accordance with the principles expressed in the Declaration of Helsinki. Study results will be published in peer-reviewed journals and presented at national and international conferences. In short, no action will be taken without written consent from patients and doctors.

Contributors PAS, LMS and JARS were involved in designing of the study. PAS was involved in writing of the manuscript. All authors read and approved the final manuscript draft.

Funding The authors have not declared a specific grant for this research from any funding agency in the public, commercial or not-for-profit sectors.

Competing interests None declared.

Patient consent None declared.

Ethics approval The Ethics Committee of the University of Beira Interior and Portuguese National Data Protection Commission.

Provenance and peer review Not commissioned; externally peer reviewed.

Open access This is an open access article distributed in accordance with the Creative Commons Attribution Non Commercial (CC BY-NC 4.0) license, which permits others to distribute, remix, adapt, build upon this work non-commercially, and license their derivative works on different terms, provided the original work is properly cited, appropriate credit is given, any changes made indicated, and the use is non-commercial. See: http://creativecommons.org/licenses/by-nc/4.0/.

\section{REFERENCES}

1. Gnjidic D, Hilmer SN, Blyth FM, et al. Polypharmacy cutoff and outcomes: five or more medicines were used to identify communitydwelling older men at risk of different adverse outcomes. J Clin Epidemiol 2012;65:989-95.

2. Maher RL, Hanlon J, Hajjar ER. Clinical consequences of polypharmacy in elderly. Expert Opin Drug Saf 2014;13:57-65.

3. Machado-Alba JE, Gaviria-Mendoza A, Machado-Duque ME, et al. Deprescribing: a new goal focused on the patient. Expert Opin Drug Saf 2017;16:111-2.

4. Ziere G, Dieleman JP, Hofman A, et al. Polypharmacy and falls in the middle age and elderly population. Br J Clin Pharmacol 2006;61:218-23.

5. Field TS, Gurwitz JH, Harrold LR, et al. Risk factors for adverse drug events among older adults in the ambulatory setting. J Am Geriatr Soc 2004;52:1349-54.
6. Bourgeois FT, Shannon MW, Valim C, et al. Adverse drug events in the outpatient setting: an 11-year national analysis. Pharmacoepidemiol Drug Saf 2010;19:901-10.

7. Jyrkkä J, Enlund H, Korhonen MJ, et al. Polypharmacy status as an indicator of mortality in an elderly population. Drugs Aging 2009;26:1039-48.

8. Scott IA, Anderson K, Freeman CR, et al. First do no harm: a real need to deprescribe in older patients. Med J Aust 2014;201:390-2.

9. Opondo D, Eslami S, Visscher S, et al. Inappropriateness of medication prescriptions to elderly patients in the primary care setting: a systematic review. PLoS One 2012;7:e43617.

10. Frank $C$. Deprescribing: a new word to guide medication review. CMAJ 2014;186:407-8.

11. Turner JP, Edwards S, Stanners M, et al. What factors are important for deprescribing in Australian long-term care facilities? Perspectives of residents and health professionals. BMJ Open 2016;6:e009781.

12. Wahab MS, Nyfort-Hansen K, Kowalski SR. Inappropriate prescribing in hospitalised Australian elderly as determined by the STOPP criteria. Int J Clin Pharm 2012;34:855-62.

13. Galazzi A, Lusignani M, Chiarelli MT, et al. Attitudes towards polypharmacy and medication withdrawal among older inpatients in Italy. Int J Clin Pharm 2016;38:454-61.

14. Qi K, Reeve E, Hilmer SN, et al. Older peoples' attitudes regarding polypharmacy, statin use and willingness to have statins deprescribed in Australia. Int J Clin Pharm 2015;37:949-57.

15. Scott IA, Hilmer SN, Reeve E, et al. Reducing inappropriate polypharmacy: the process of deprescribing. JAMA Intern Med 2015;175:827-34.

16. Jansen J, Naganathan V, Carter SM, et al. Too much medicine in older people? Deprescribing through shared decision making. BMJ 2016;353:i2893.

17. Reeve E, Shakib S, Hendrix I, et al. Review of deprescribing processes and development of an evidence-based, patient-centred deprescribing process. Br J Clin Pharmacol 2014;78:738-47.

18. Whitman AM, DeGregory KA, Morris AL, et al. A Comprehensive Look at Polypharmacy and Medication Screening Tools for the Older Cancer Patient. Oncologist 2016;21:723-30.

19. Page AT, Clifford RM, Potter K, et al. The feasibility and effect of deprescribing in older adults on mortality and health: a systematic review and meta-analysis. Br J Clin Pharmacol 2016;82:583-623.

20. Beer C, Loh PK, Peng YG, et al. A pilot randomized controlled trial of deprescribing. Ther Adv Drug Saf 2011;2:37-43.

21. Reeve E, Shakib S, Hendrix I, et al. The benefits and harms of deprescribing. Med J Aust 2014;201:386-9.

22. Ryan R, Hill S. Making rational choices about how best to support consumers' use of medicines: a perspective review. Ther Adv Drug Saf 2016;7:159-64.

23. Gnjidic D, Le Couteur DG, Kouladjian L, et al. Deprescribing trials: methods to reduce polypharmacy and the impact on prescribing and clinical outcomes. Clin Geriatr Med 2012;28:237-53.

24. Duerden M, Avery T, Payne R. Polypharmacy and medicines optimisation Making it safe and sound. Kings Fund [Internet] 2013:1-68 http://www.kingsfund.org.uk/publications.

25. Stiggelbout AM, Van der Weijden T, De Wit MP, et al. Shared decision making: really putting patients at the centre of healthcare. $B M J$ 2012;344:e256.

26. Reeve E, Wiese MD, Hendrix I, et al. People's attitudes, beliefs, and experiences regarding polypharmacy and willingness to Deprescribe. J Am Geriatr Soc 2013;61:1508-14.

27. Reeve E, To J, Hendrix I, et al. Patient barriers to and enablers of deprescribing: a systematic review. Drugs Aging 2013;30:793-807.

28. Batel-Marques F, Mendes D, Alves C, et al. [Pharmacovigilance in Portugal: Activity of the Central Pharmacovigilance Unit]. Acta Med Port 2015;28:222-32.

29. Prazeres F, Santiago L. Prevalence of multimorbidity in the adult population attending primary care in Portugal: a cross-sectional study. BMJ Open 2015;5:e009287. 